\title{
Near field phased array DOA and range estimation of UHF RFID tags
}

\author{
Jordy Huiting, André B.J. Kokkeler and Gerard J.M. Smit \\ Dep. of Electrical Engineering, Mathematics and Computer Sciencem, University of Twente, Enschede \\ Email: j.huiting@utwente.nl
}

\begin{abstract}
This paper presents a near field localization system based on a phased array for UHF RFID tags. To estimate angle and range the system uses a two-dimensional MUSIC algorithm. A four channel phased array is used to experimentally verify the estimation of angle and range for an EPC gen2 tag. The system is calibrated for phase offsets introduced by hardware as simulations show the sensitivity to these offsets. Experiments with this calibrated receiver array give inaccurate ranging estimates. A solution is to calibrate the system for every angle, allowing meaningful range estimates. Experiments in a different environment show a reduced result, indicating the need for extensive calibration.
\end{abstract}

\section{INTRODUCTION}

RFID tags are more and more used to replace bar codes. The main advantages are non line of sight and long read range in combination with more data storage. EPC Gen2 tags, operating in the UHF band, can be used to identify products individually instead of group based barcodes at a relatively low cost, especially when using batteryless passive tags. This enables the tracking and localization of individual objects. With a typical portal like reader, presence near the portal can be concluded from reading a tag. Several attempts have been made to localize a tag more precisely. With the help of a phased array it is possible to determine the direction of arrival of a tag signal. Traditionally the signal is assumed to originate from the far field and only a direction can be estimated. When multiple antennas are used as a phased array however, the read range of UHF RFID is small compared to the size of the array. Therefor the far field model can not be completely justified. This paper tries to exploit the near field model in combination with a four channel phased array to estimate an angle and range of a nearby tag.

\section{EPC GEN2 PROTOCOL}

The EPC Gen 2 procotol defines the communication between a tag and reader in the UHF frequency band. The system uses a reader-talks-first algorithm and selects an available tag randomly. A passive tag within range of the reader is powered by the transmitted signal and at the same time receives commands and data from the reader. The selected tag starts transmitting requested data by modulating the continuous wave transmitted by the reader. This backscattered signal is received by the reader and demodulated to retrieve the requested data, which can include the identifying number, the Electronic Product Code (EPC). In a conventional setup, this backscatter is received by a single antenna. This can be the same antenna transmitting the continuous wave (known as a monostatic setup), or a separate antenna (a bi-static setup). In the setup presented in this paper the signals are received by multiple antennas placed in line to form a phased array [1].

\section{A. Far field angle of arrival estimation}

In the context of localization of EPC Gen2 RFID tags, the use of phased arrays is proposed to estimate an angle of arrival. For example [2] uses four phased arrays consisting of three antennas each, located on corners of a square to localize tags within the square. A disadvantage of this technique is the requirement to use multiple antenna arrays, the system proposed in this paper tries to use only a single antenna array. [3] uses a circular, 8 antenna array to estimate the angle of arrival in two dimensions using the MUSIC algorithm. These algorithms estimate an angle of arrival and assume far field conditions. However, in relation to the dimensions of the phased array, there is no clear separation between the near and far-field. This paper assumes the near field model with respect to the array to be able to estimate the range and angle.

\section{SIgNAL MODEL}

The signal backscattered by the tag $s(t)$ can be seen as arriving from a certain angle $\theta$ at the array. When satisfying the far field conditions, the signals arrive as a plane wave at the array. When the source is located in the near field of the receiving antenna, the signals no longer arrive as a plane wave. The backscattered signal propagates allong a sphere and is sampled by the array. Compared to the sampling of a plane wave there is some additional phase difference depending on the range. Although there is a gradual transition between near and far-field, a commonly used distance to separate the near and far-field is the Fraunhofer distance $R_{\text {farfield }}=\frac{2 D^{2}}{\lambda}$ whereby $D$ is the largest dimension of the antenna and $\lambda$ the wavelength [4]. An array of antennas can be seen as one antenna in this formula. Assuming 4 antennas spaced $0.5 \lambda$ apart at UHF RFID frequencies, this range is about 1.5 meters. When the size of the array is increased to 6 antennas the near field extents to more than 4 meters.

The distance between a tag and an individual antenna of the array can be derived from Figure 1 .

$$
\begin{array}{rrr}
r_{m} & = & \sqrt{(r \cos (\theta))^{2}+(\sin (\theta) r-M d)^{2}} \\
& =\sqrt{r^{2}+(M * d)^{2}-2 * \sin (\theta) * r * M * d}
\end{array}
$$

whereby $m$ indicates the number of antenna being used and $d$ resembles the spacing between the antennas. $M$ indicates the distance from the center of the array, when the antenna index $m$ starts counting at $0, M$ is defined as $\left(m-\frac{m}{2}\right)$. The bandwidth of the information signal, with a maximum 


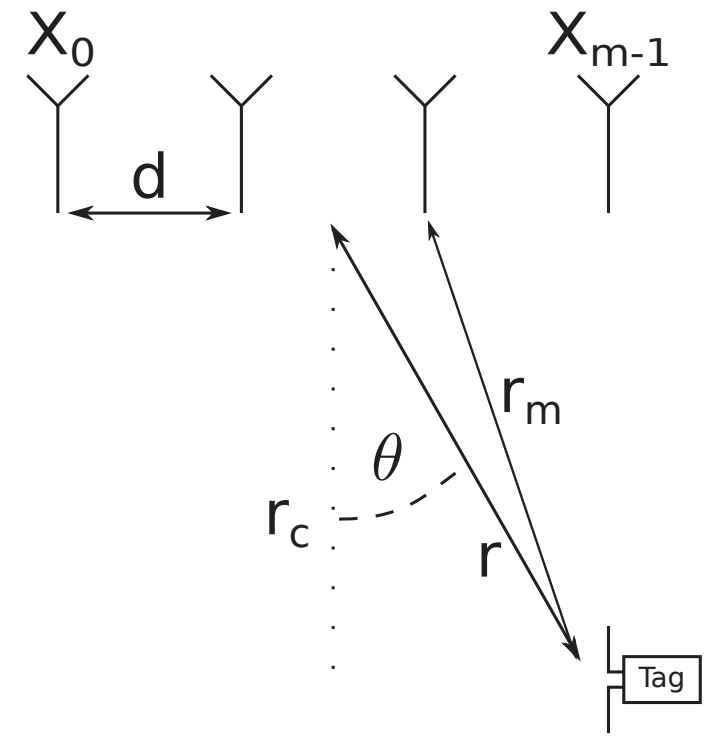

Fig. 1. Near field model

bandwidth of $200 \mathrm{kHz}$ [1], modulated on a carrier of $865 \mathrm{MHz}$ justifies the narrowband assumption. Therefor the shift of the signal in time due to distance can be modeled as a phase shift [5]. Besides a phase shift, the distance between the tag and receiving antenna will influence the amplitude of the received signal. For now this dependence is not included in the model. The signal $X_{m}(t)$ received by the $m$ th antenna is modeled as:

$$
X_{m}(t)=\sum_{i=1}^{N} s_{i}(t) * e^{j \frac{2 \pi}{\lambda} * r_{m}}+n_{m}(t)
$$

In this equation $N$ resembles the number of uncorrelated signal sources which arrive from different angles and ranges. In addition to the distance between the receiving antennas and the tag, the distance between the antenna used for transmitting and the tag will have a phase changing effect. This effect can be modeled in a similar fashion and added as an extra term in (1).

The received signals, $X_{m}$, can be combined in the vector $\mathbf{X}(t)$. The $N \mathrm{x} m$ matrix $\mathbf{A}$ consists of the exponential phase terms. The additive white noise can be combined in the noise vector $\mathbf{n}(t)$.

$$
\mathbf{X}(t)=\mathbf{A} s(t)+\mathbf{n}(t)
$$

With a far field mode,l only the direction of arrival (DOA) of the arriving signals can be estimated. The far field model has been extensively studied and numerous direction of arrival estimation methods exist. Assuming near field conditions, not only the angle but also the distance between the tag and antenna array can be estimated. The goal of this paper is to estimate these parameters with a linear array. The 2dimensional variant of the MUSIC algorithm will be used in simulations and experiments.

\section{2D RANGE AND DOA ESTIMATION}

Range and DOA estimation based on the near field assumption has received some interest. The traditional DOA estimation techniques like MUSIC and ESPRIT have been transformed into $2 \mathrm{~d}$ variants. Although a $2 \mathrm{~d}$ variant of the MUSIC algorithm is straight forward [6], it results in a computationally intensive $2 \mathrm{~d}$ search. To enable fast computation this search can be parallelized. The $2 \mathrm{~d}$ variant of ESPRIT requires higher order statistics and is also computationally demanding [7]. Furthermore, computationally efficient algorithms have been derived [8]. The MUSIC algorithm is known for it high resolution and robustness against phase deviations [9] and is therefor used in the following experiments, the high computational load is of lesser concern due to offline processing.

\section{A. MUSIC Algorithm}

The one-dimensional MUSIC algorithm is based on the fact that signal and noise subspace are orthogonal [10]. A covariance matrix is calculated and split into signal and noise subspaces by means of an eigendecomposition. Instead of focussing on the signal subspace as ESPRIT does, the MUSIC algorithm uses the noise subspace to find the signal parameters. The covariance matrix is of size $m \mathrm{xm}$, by assuming the reception of only a single signal, the noise subspace $\mathbf{E}_{n}$ is of size $m-1 \mathrm{x} m$. The received signal is modeled to construct steering vectors, a, which should be orthogonal to the noise subspace. The algorithm searches through all possible steering vectors, $\mathbf{a}(\theta)$ for a far field model, and $\mathbf{a}(\theta, r)$ for the near field model. $\mathbf{a}(\theta, r)$ is modeled after equation 3 . The algorithm tries to find the steering vector(s) that are the most orthogonal to the noise subspace. For the far field model this leads to

$$
\theta_{\text {est }}=\max _{\theta} \frac{1}{\mathbf{a}(\theta)^{H} \mathbf{E}_{n} \mathbf{E}_{n}^{H} \mathbf{a}(\theta)}
$$

The Euclidian distance from a vector to a signal is zero if they are orthogonal. Calculating the squared Euclidian distance $\mathbf{a}(\theta)^{H} \mathbf{E}_{n} \mathbf{E}_{n}^{H} \mathbf{a}(\theta)$ for a range of angles gives a small outcome when the steering vector $a(\theta)$ is orthogonal to the noise subspace. The MUSIC pseudo-spectrum is defined as the inverse of this search, see (4). To estimate not only angle but also range, the model has to be changed. Therfore the steering vector is changed to $\mathbf{a}(\theta, r)$, based on the model described in section III. A two dimensional search is then used to estimate the angle and range.

$$
\left(\theta_{\text {est }}, r_{\text {est }}\right)=\max _{\theta, r}\left(\frac{1}{\mathbf{a}(\theta, r)^{H} \mathbf{E}_{n} \mathbf{E}_{n}^{H} \mathbf{a}(\theta, r)}\right)
$$

\section{Simulations}

Simulations are conducted to verify the ability of the MUSIC algorithm to estimate angle and range. An Additive White Gaussian Noise (AWGN) channel is used for these simulations, with a resulting signal to noise ratio of $21 \mathrm{~dB}$ per communication channel, to simulate a realistic environment [11]. Only four antennas are simulated because the intended hardware setup for experiments uses the same number of antennas. The number of samples used to calculate a single range estimation is chosen to be 2048, again simulating the proposed hardware.

In Figure 2 the simulation results of ranging estimates to a single tag are shown. Furthermore the array is being simulated with and without phase and gain offset. Every receiver chain is expected to have the same influence on the received signal, 


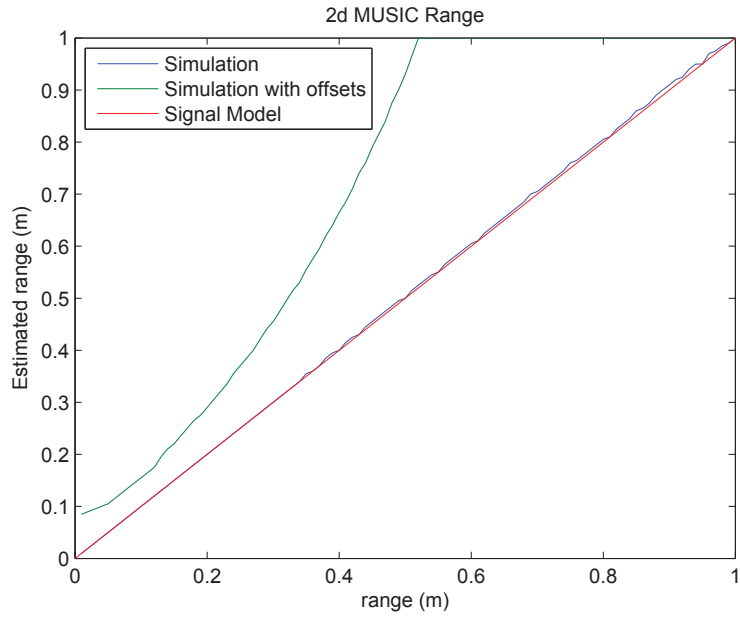

Fig. 2. Simulation results: 2d MUSIC range estimation

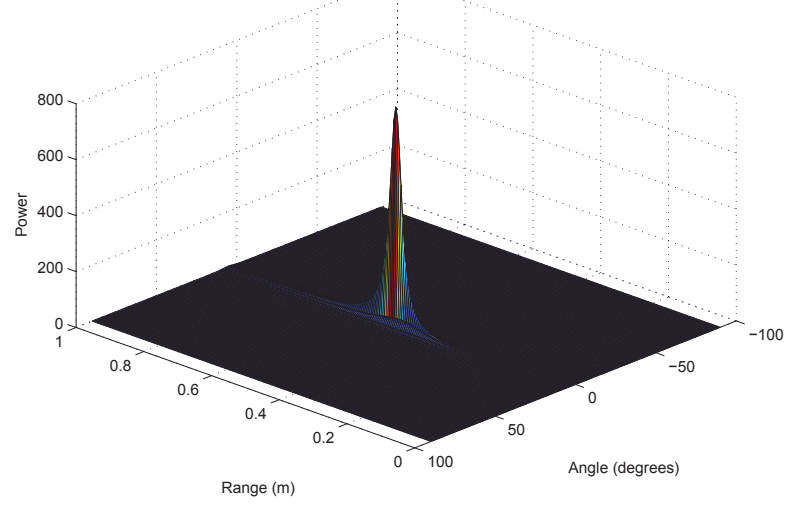

Fig. 3. Simulation results: 2d MUSIC pseudospectrum

however in a real system the analog filters and wires might introduce a different phase shift and attenuation. These phase and gain offsets introduced by hardware tolerances can add up and for this simulation the measured values from table I are used. The $2 \mathrm{~d}$ variant of the MUSIC algorithm is able to estimate the range to a tag if the phase and gain offsets are known. In Figure 3 the resulting (pseudo) spectrum of the $2 \mathrm{~d}$ MUSIC algorithm is shown with a peak indicating the estimated range and angle of the simulated tag.

\section{EXPERIMENTS}

A four channel receive array is used for experiments. Each channel consists of a patch antenna, Low Noise Amplifier (LNA), downconverter and 14-bit ADC. In correspondence with the simulations, the antennas are spaced $0.5 \lambda$ apart, the same values as used for simulations. Furthermore anti aliasing filters are present in the receiver chains. The digital baseband information is fed into a Digital Signal Processing (DSP) board and recorded. The Gen2 tags are exited by an Impinj R2000 based, off the shelf reader [12]. To prevent influence of the default hopping behavior, the R2000 is set to use a single frequency. The R2000 communicates normally with the tags

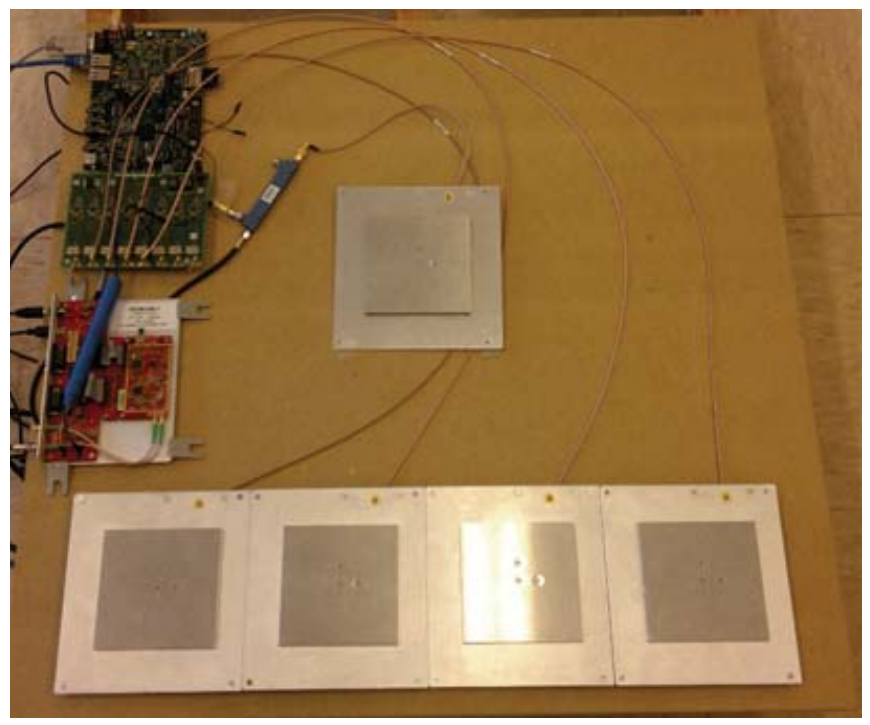

Fig. 4. EPC gen2 reader and 4 channel phased array hardware

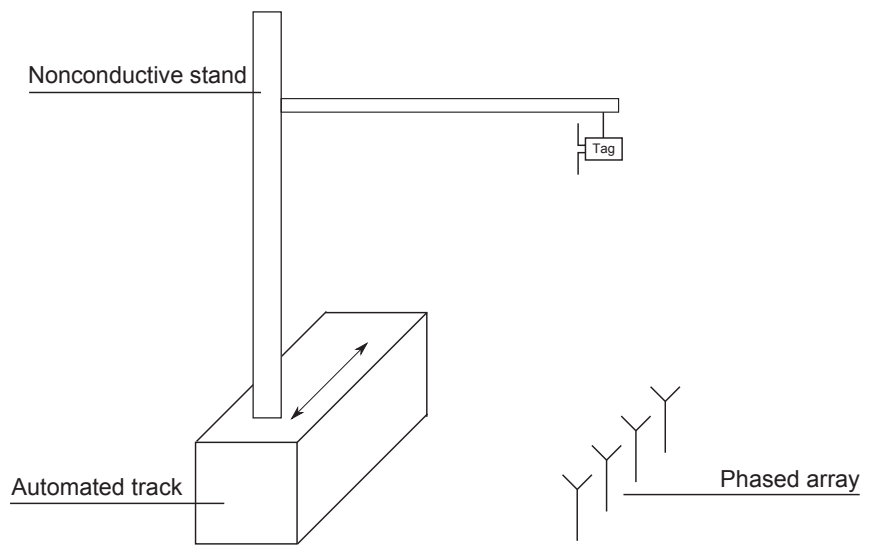

Fig. 5. Automated test setup

via a single separate antenna. The continuous wave transmitted by the R2000 during tag to reader communication is used by the downconverters of the 4 array reception channels. The sampling frequency of the ADC is fixed at $1200 \mathrm{kHz}$. This sampling frequency is well beyond the Nyquist rate of the baseband signal and is chosen to place aliases of nearby readers out of band. Of every (successful) tag to reader communication period, 2048 stored samples are transfered to a PC for offline processing.

Every channel of the receiver is designed equally, however small deviations can add up and introduce phase and gain errors in the form of a bias. To calibrate the system, measurements with consistent distance and angle are used to calculate average phase and gain offsets for every channel. These offsets are shown in table I and are assumed to be caused by hardware tolerances.

\section{TABLE I. PHASE AND GAIN OFFSETS}

\begin{tabular}{|l|l|l|l|l|}
\hline Antenna & 1 & 2 & 3 & 4 \\
\hline Phase offset $(\mathrm{rad})$ & -0.3 & -0.7 & -0.4 & 0 \\
\hline Gain offset & 1.4 & 1.6 & 0.9 & 1.0 \\
\hline
\end{tabular}




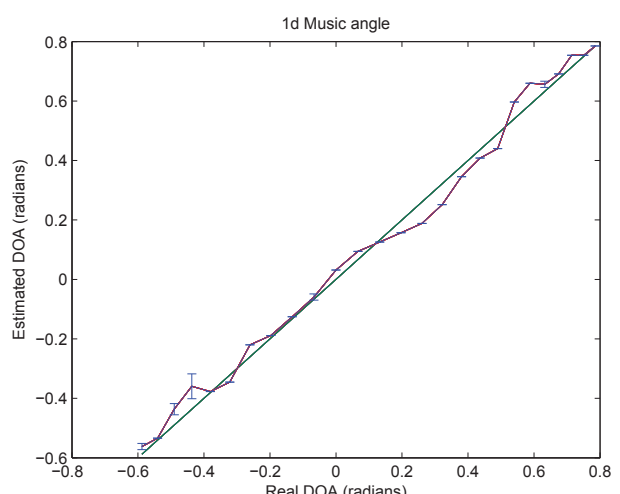

Fig. 6. 1d MUSIC doa estimation

\section{A. Automated test setup}

A gen2 tag is placed on an automated test rig consisting of a computer controlled servo, aluminium track and nonconductive stand, as shown in Figure 5. The tag is moved horizontally in discrete steps by the servo. At every stop, the rfid reader starts interrogating the tag and ADC samples from 200 successful tag reads are stored. After a successful run of the servo over the entire horizontal range, the tag has to be manually moved in vertical direction to measure at a different distance with respect to the array.

\section{B. One- and Two-dimensional MUSIC}

To verify the experimental setup, the one dimensional MUSIC algorithm is used to estimate the direction of arrival. In Figure 6 the results are shown. This figure shows the average and standard deviation of a measurement run with a 0.75 meter distance between the tag and the center of the array, $r_{c}$ of Figure 1. Furthermore it clearly shows a positive relation between the average estimated angle and the expected angle. Besides estimating an angle, the MUSIC algorithm can be used to estimate a range, as shown by simulations. In Figure 7 a range estimation based on experimental data is shown for the same data as in Figure 6. The 2d MUSIC algorithm is not able to correctly determine the range. For a single measurement position of the tag, the phase deviation between multiple measurements is small as shown by the error bars for an individual measurement position. There are two possible explanations for these large errors, one possible explanation for this behavior is an angle dependent phase shift caused by each individual antenna, as is also found in [13]. In a far field scenario this angle dependent phase shift has no influence on the estimated angle because a linear wave front is assumed, thus arriving at the array antennas with only an angle dependent phase shift. In the near field model however, the signal arrives at every antenna with a different angle. Therefor the phase dependent behavior has to be taken into account. In the following section calibration for this angle dependent phase shift is elaborated. Another possibility is that the system is disturbed by the environment, the presence of fine grained reflections can cause indistinguishable phase shifts at the receive antennas. It is known that the UHF RFID system can be severely influenced by reflections [14].

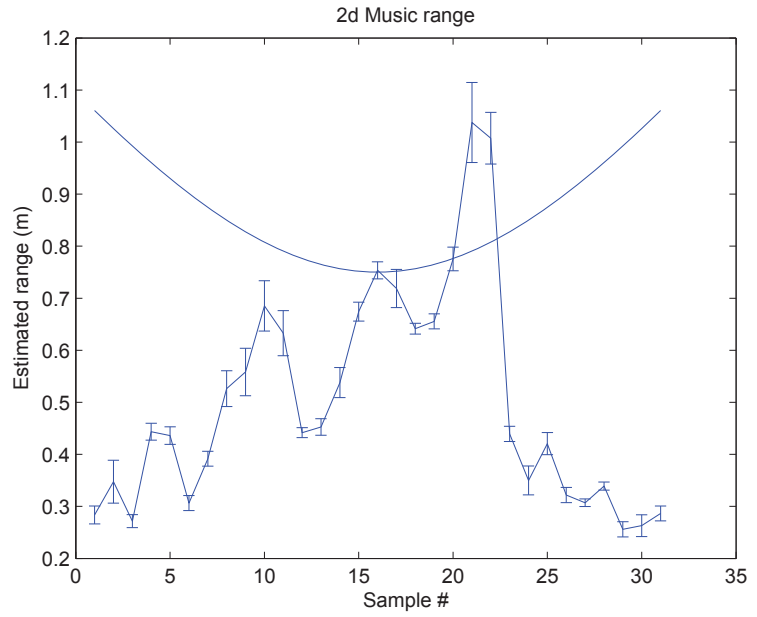

Fig. 7. Normal calibrated range

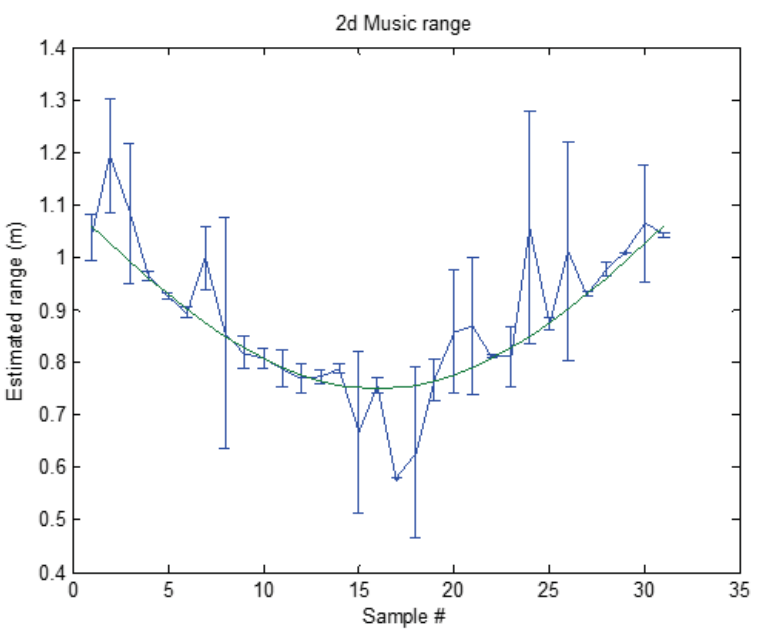

Fig. 8. Calibrated range

\section{Angle dependent calibration}

The received phase appears to be influenced by effects that are not modeled. To test the effect of correct signal propagation modeling, the first sample of every measurement position is used to calibrate the array. In a practical system this would translate into calibration for every change in environment.

As can be seen in Figure 8 this system is able to determine the range more accurately than the uncalibrated system.

It is expected that fine grained reflections cause this need for calibration. In a different environment the reflections will be different, so the calibration process has to be repeated. To show the need for recalibration the same experiments are done in a different (smaller) room. The calibration data from the large empty room used above are applied on the measurement data. As can bee seen in Figure 9 by reusing calibration in a different room, the estimation of range is inaccurate. This shows the influence of the environment on the proposed algorithm. 


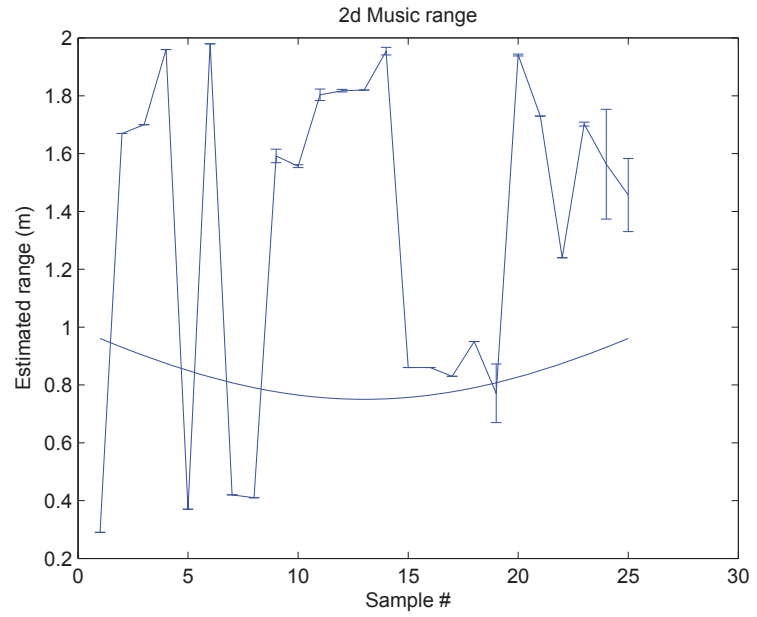

Fig. 9. Reusing calibration data to estimate range

\section{CONCLUSION}

The far field assumption can be invalid when a four channel phased is used to interrogate EPC gen2 tags. This is certainly true when the range between the tag and array is small, for example in a portal setup. By using a four channel phased array and calibrating the measured phases for every angle experiments have shown that it is possible to determine the direction of arrival and range by means of the $2 \mathrm{~d}$ MUSIC in the near field region of a phased array. This calibration process has to be repeated for every change in measurement environment. Such a process is impractical for most applications as the environment may change over time and would require recalibration.

\section{ACKNOWLEDGMENT}

This paper describes work in part undertaken in the context of the TSP project. The TSP project has joint funding from the European Regional Development Fund of the European Union and the Dutch Provinces of Gelderland and Overijssel.

\section{REFERENCES}

[1] D. M. Dobkin, The RF in RFID: Passive UHF RFID in Practice. Newnes, 2008.

[2] S. Azzouzi, M. Cremer, U. Dettmar, R. Kronberger, and T. Knie, "New measurement results for the localization of UHF RFID transponders using an Angle of Arrival (AoA) approach," in RFID (RFID), 2011 IEEE International Conference on, April 2011, pp. 91-97.

[3] G. Hislop and C. Craeye, "Spatial smoothing for $2 \mathrm{~d}$ direction finding with passive RFID tags," in Antennas Propagation Conference, 2009. LAPC 2009. Loughborough, Nov 2009, pp. 701-704.

[4] C. A. Balanis, Antenna Theory: Analysis and Design. Newnes, 1997.

[5] B. Allen and M. Ghavami, Adaptive Array Systems, Fundamentals and Applications. John Wiley \& Sons, 2005.

[6] Y.-D. Huang and M. Barkat, "Near-field multiple source localization by passive sensor array," Antennas and Propagation, IEEE Transactions on, vol. 39, no. 7, pp. 968-975, Jul 1991.

[7] R. Challa and S. Shamsunder, "High-order subspace-based algorithms for passive localization of near-field sources," in Signals, Systems and Computers, 1995. 1995 Conference Record of the Twenty-Ninth Asilomar Conference on, vol. 2, Oct 1995, pp. 777-781 vol.2.
[8] N. Tayem, C. Attanaayake, and A. Abatan, "Range and Bearing Estimation for Near-Field Sources," in Vehicular Technology Conference Fall (VTC 2010-Fall), 2010 IEEE 72nd, Sept 2010, pp. 1-5.

[9] R. Roy and T. Kailath, "ESPRIT-estimation of signal parameters via rotational invariance techniques," Acoustics, Speech and Signal Processing, IEEE Transactions on, vol. 37, no. 7, pp. 984-995, Jul 1989.

[10] R. Schmidt, "Multiple emitter location and signal parameter estimation," Antennas and Propagation, IEEE Transactions on, vol. 34, no. 3, pp. 276-280, Mar 1986.

[11] P. Nikitin and K. Rao, "Antennas and Propagation in UHF RFID Systems," in RFID, 2008 IEEE International Conference on, April 2008, pp. 277-288.

[12] Indy R2000 Reader Chip, Impinj, 1 2014, rev. 1.4.

[13] R. Miesen, F. Kirsch, and M. Vossiek, "UHF RFID Localization Based on Synthetic Apertures," Automation Science and Engineering, IEEE Transactions on, vol. 10, no. 3, pp. 807-815, July 2013.

[14] D. Arnitz, U. Muehlmann, and K. Witrisal, "Characterization and Modeling of UHF RFID Channels for Ranging and Localization," Antennas and Propagation, IEEE Transactions on, vol. 60, no. 5, pp. 2491-2501, May 2012. 